(6) OPEN ACCESS

\title{
Minimum reporting standards for clinical research on groin pain in athletes
}

\author{
Eamonn Delahunt, ${ }^{1,2}$ Kristian Thorborg, ${ }^{3}$ Karim M Khan, ${ }_{1}^{4}$ Philip Robinson, ${ }^{5}$ \\ Per Hölmich, ${ }^{3,4}$ Adam Weir ${ }^{4}$
}

${ }^{1}$ School of Public Health, Physiotherapy and Population Science, University College Dublin, Dublin, Ireland

${ }^{2}$ Institute for Sport and Health, University College Dublin,

Dublin, Ireland

${ }^{3}$ Sports Orthopedic Research

Center-Copenhagen (SORC-

C), Arthroscopic Center

Amager, Department of

Orthopaedic Surgery,

Copenhagen University

Hospital, Amager-Hvidovre, Denmark

${ }^{4}$ Aspetar Sports Groin Pain Center, Aspetar Orthopaedic and Sports Medicine Hospital, Doha, Qatar

${ }^{5}$ Leeds Musculoskeletal Biomedical Research Unit, Leeds Teaching Hospitals, University of Leeds, Leeds, UK

\section{Correspondence to}

Dr Eamonn Delahunt, School

of Public Health, Physiotherapy and Population Science,

University College Dublin, Health Sciences Center, Belfield, Dublin 04, Ireland; eamonn.delahunt@ucd.ie

Accepted 10 April 2015

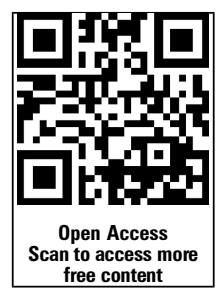

CrossMark

To cite: Delahunt $\mathrm{E}$,

Thorborg K, Khan KM, et al. $\mathrm{Br}$ J Sports Med

2015;49:775-781.

\section{ABSTRACT}

Groin pain in athletes is a priority area for sports physiotherapy and sports medicine research. Heterogeneous studies with low methodological quality dominate research related to groin pain in athletes. Lowquality studies undermine the external validity of research findings and limit the ability to generalise findings to the target patient population. Minimum reporting standards for research on groin pain in athletes are overdue. We propose a set of minimum reporting standards based on best available evidence to be utilised in future research on groin pain in athletes. Minimum reporting standards are provided in relation to: (1) study methodology, (2) study participants and injury history, (3) clinical examination, (4) clinical assessment and (5) radiology. Adherence to these minimum reporting standards will strengthen the quality and transparency of research conducted on groin pain in athletes. This will allow an easier comparison of outcomes across studies in the future.

\section{INTRODUCTION}

Groin pain is common in athletes participating in multidirectional team sports. In a recent review, Orchard ${ }^{1}$ reported that ice hockey and football codes (other than soccer) involving twisting/turning and kicking have high rates of groin injuries. Additionally, Waldén, et $a l^{2}$ reported that groin injuries are common in senior soccer. Groin pain in athletes is a considerable challenge for clinicians owing to its high incidence and prevalence, association with significant time loss from sport and high recurrence rates. It is an important area for sports physiotherapy and sports medicine research.

A recent systematic review on the treatment of groin pain in athletes identified 72 studies. $^{3}$ Only four of the studies were high quality, and there was an inverse correlation between the reported outcome and the methodological quality score. Low methodological quality frequently resulted from a lack of basic information such as the absence of a suitable control group, failure to conceal allocation and failure to blind either study participants, assessors or those involved in the treatment. The review also highlighted the confusion surrounding the nomenclature of groin pain in athletes, as 33 different diagnostic terms were used in the 72 studies. ${ }^{3}$ Furthermore, inadequate reporting of participant characteristics, injury history and clinical examination findings was common. Heterogeneous taxonomy and confusion in the field motivated the "Doha agreement meeting on terminology and definitions in groin pain in athletes"; ${ }^{4}$ this consensus paper proposes a clinical classification system and uniform terminology for groin pain in athletes. The
Doha agreement also identified the need to provide minimum methodological and clinical reporting standards for research on groin pain in athletes. ${ }^{4}$ This would improve the potential for data synthesis for systematic reviews and meta-analyses.

In both research and clinical practice, MRI is increasingly being used for the assessment of groin pain in athletes. However, in a recent review, Branci et $a l^{5}$ concluded that the methodological quality of imaging studies remains low. Common methodological limitations include inconsistent diagnostic terminology, the regular inclusion of heterogeneous study populations and a lack of reliable assessment procedures.

Considering the aforementioned fairly fundamental limitations, the aim of the present paper was to provide minimum reporting standards in five areas of research on groin pain in athletes. These specific areas include: (1) study methodology, (2) study participants and injury history, (3) clinical examination, (4) clinical assessment and (5) radiology.

\section{METHODS}

\section{Design}

This paper was initiated by the primary author (ED), in conjunction with researchers (AW and $\mathrm{PH}$ ) from the Sports Groin Pain Center, Aspetar Orthopaedic and Sports Medicine Hospital, Doha, Qatar. An additional three international experts (KT, KMK and PR) were invited to participate. The experts were selected for their expertise, according to the interpretation of the initiating researchers, and they did not represent specific organisations.

\section{Procedure}

The primary author was previously involved in the development of a consensus statement related to chronic ankle instability (CAI), ${ }^{6-8}$ and thus had experience in developing guidelines relating to minimum reporting standards. The key items for inclusion in the present paper were based on the collective expertise of the group along with analysis of key methodological shortcomings of published research in the area identified by recent systematic reviews. $^{3} 5$ Collectively, the group agreed that minimum reporting standards could be improved in relation to: (1) study methodology, (2) study participants and injury history, (3) clinical examination, (4) clinical assessment and (5) radiology.

\section{RESULTS}

Minimum reporting standards on study methodology

The credibility of research is dependent on the ability of end users to critically assess the study 
Table 1 Published guidelines to improve study methodological design and reporting

$\begin{array}{ll}\text { Study design } & \text { Recommended guideline } \\ \text { RCT } & \text { CONSORT checklist and guide }{ }^{9} \\ \text { Non-randomised intervention } & \text { TREND statement }{ }^{10} \\ \text { Cohort } & \text { STROBE statement }{ }^{11} \\ \text { Case-control } & \text { STROBE statement }{ }^{11} \\ \text { Cross-sectional } & \text { STROBE statement }{ }^{11}\end{array}$

CONSORT, Consolidated Standards of Reporting Trials; RCT, randomised controlled trial; STROBE, Strengthening the Reporting of Observational Studies in Epidemiology; TREND, Transparent Reporting of Evaluations with Nonramdomised Designs.

design, conduct and analysis. Therefore, for future research on groin pain in athletes, we recommend using established published guidelines to improve study methodological design and reporting (table 1$)$.

For researchers designing clinical trials, we recommend using the Standard Protocol Items: Recommendations for Intervention Trials (SPIRIT) 2013 statement, ${ }^{12}$ which provides a guide for preparing clinical trial protocols. Furthermore, in accordance with the recommendations of the International Committee of Medical Journal Editors (ICMJE), ${ }^{13}$ we recommend that researchers should consider registering their trial in any registry that is a primary register of the WHO International Clinical Trials Registry Platform, ${ }^{14}$ or in ClinicalTrials.gov. ${ }^{15}$ The purpose of clinical trial registration is to prevent the selective publication and reporting of research outcomes. With reference to the reporting of randomised controlled trials (RCTs), we recommend using the Consolidated Standards of Reporting Trials (CONSORT) 2010 statement, ${ }^{9}$ which was developed to improve the quality of RCT reporting. When reporting on a non-randomised intervention, we recommend that authors use the Transparent Reporting of Evaluations with Nonrandomized Designs (TREND) statement. ${ }^{10}$ For researchers reporting observational studies, including cohort, case-control and cross-sectional studies, we recommend using the Strengthening The Reporting of Observational Studies in Epidemiology (STROBE) statement guidelines. ${ }^{11}$

The evaluation of interventions is of primary importance to clinicians and researchers alike. Without a transparent and comprehensive published description of an intervention, the utility of the intervention may be compromised. As such, for those research studies investigating the efficacy of a specific intervention, we endorse compliance with the Template for Intervention Description and Replication (TIDieR) checklist and guide. ${ }^{16}$ With reference to RCTs, the TIDieR checklist and guide should be used concomitantly with the CONSORT checklist. Furthermore, for observational studies, the TIDieR checklist and guide should be used to supplement the STROBE statement.

Key methodological considerations for the design and reporting of studies are outlined in figure 1.

\section{Minimum reporting standards on study participants and injury history}

A comprehensive and transparent description of the eligibility criteria used to select study participants is essential, and should always be reported. Clearly defined eligibility criteria are central to a study's external validity, allowing readers to interpret its applicability and relevance to their clinical practice. The following demographic information of the participants should always be reported: (1) sex; (2) mean age; (3) mean body mass; (4) mean height; and (5) sport and level participated in. Additionally, injury history should be documented for each study participant. This includes: (1) mechanism of injury (eg, acute trauma, defined mechanism, insidious onset); (2) location of symptoms; (3) duration of symptoms; and (4) validated questionnaire score to describe the current level of injury-associated disability. Regarding the location of symptoms, we recommend that researchers outline the exact location of the athlete's recognisable pain. The Copenhagen Hip and Groin Outcome Score (HAGOS) could be used to quantify an athlete's current level of injury-associated disability as it is valid, reliable and has established discriminative capacity. ${ }^{17}$

Key considerations for reporting on study participants and injury history are outlined in figure 2 .

\section{Minimum reporting standards on clinical examination}

Clinical examination findings in study participants should be thoroughly described. Researchers should try to use clinical examination methodologies that are standardised and reliable. Additionally, when choosing clinical examination methodologies, researchers should also consider their discriminative capacity. At a minimum, we recommend that researchers comprehensively describe the clinical examination
Figure 1 Considerations for the design and reporting of studies on groin pain in athletes. CONSORT, Consolidated Standards of Reporting Trials; RCT, randomised controlled trial; STROBE, Strengthening the Reporting of Observational Studies in Epidemiology; SPIRIT, Standard Protocol Items: Recommendations for Intervention Trials; TIDieR, Template for Intervention Description and Replication; TREND, Transparent Reporting of Evaluations with Nonrandomised Designs.

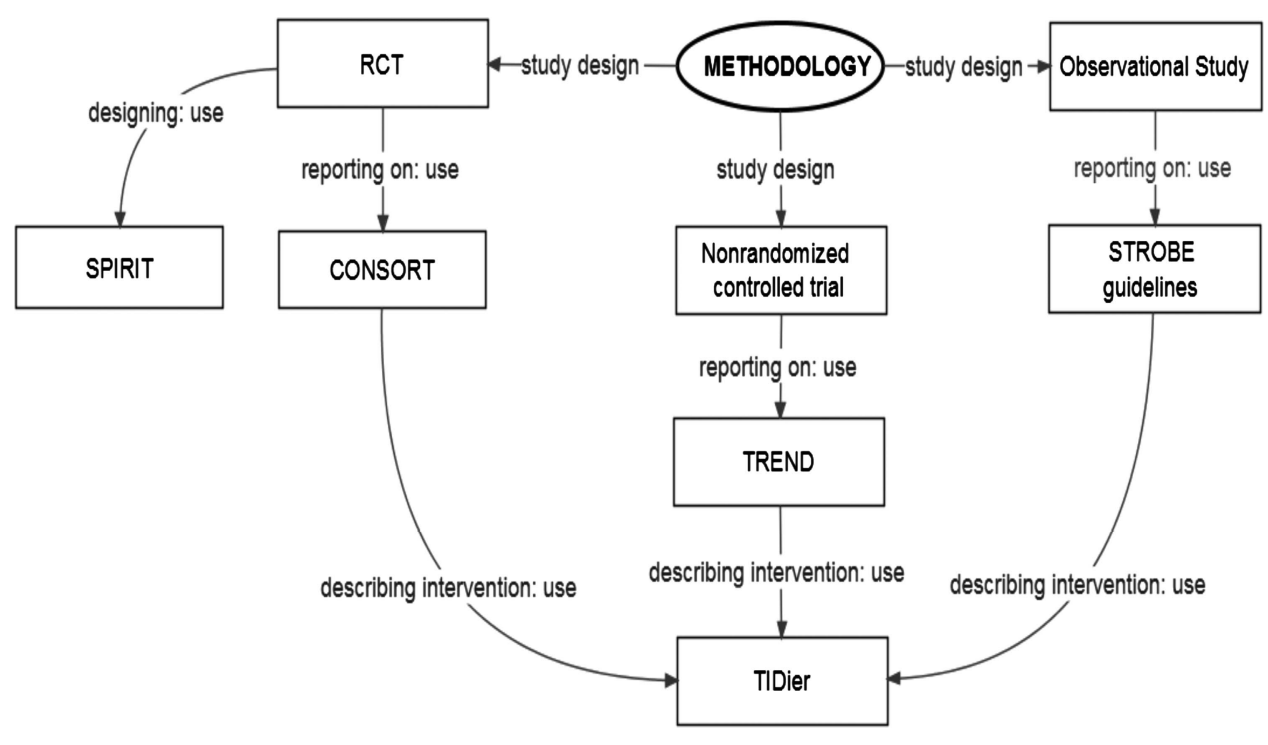

Delahunt E, et al. Br J Sports Med 2015;49:775-781. doi:10.1136/bjsports-2015-094839 
Figure 2 Considerations for the reporting on participants and injury history in studies on groin pain in athletes.

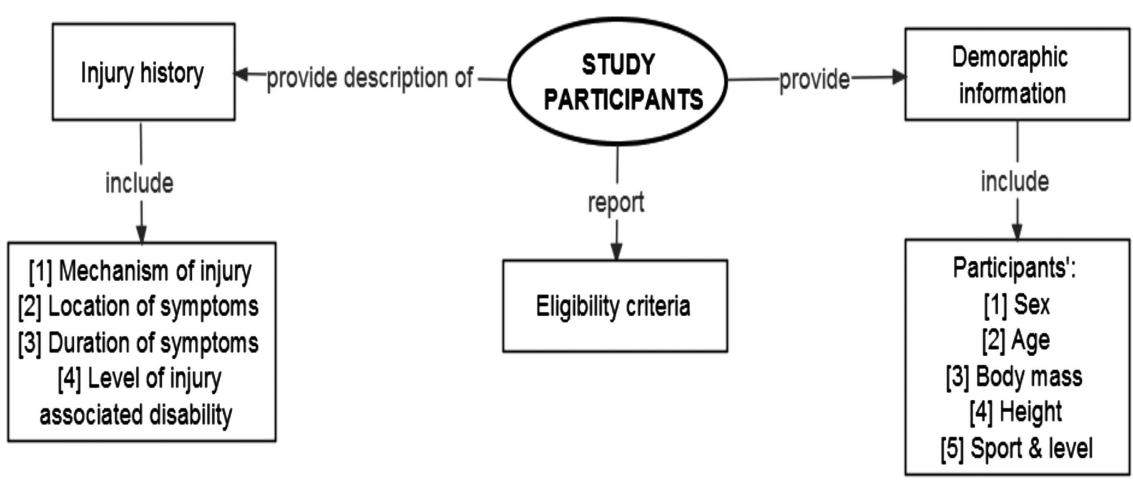

methodologies used, and associated diagnostic criteria, so that others can reproduce them. Key considerations for reporting on clinical examination are outlined in figure 3.

The "Doha agreement meeting on terminology and definitions in groin pain in athletes" reports a clinical classification system for athletes with groin pain. ${ }^{4}$ The system has three major subheadings: (1) defined clinical entities for groin pain in athletes (adductor-related, iliopsoas-related, inguinal-related, pubicrelated); (2) hip-related groin pain in athletes and (3) other causes of groin pain in athletes. Clinical examination reporting standards relative to (1) and (2) above are discussed below.

\section{Adductor-related groin pain}

Adductor-related groin pain is defined by tenderness on palpation of the adductors as well as pain on resisted hip adduction. Tenderness is defined as discomfort or pain when the area is palpated and whereby the athlete recognises this to be associated with their specific injury pain. The pain on resisted adduction testing should reproduce the athlete's recognisable pain in the adductors. As tenderness on palpation and pain on resistance testing are essential for diagnosing this entity, we recommended that during clinical examination the following are noted and subsequently reported: (1) presence of tenderness on palpation of the adductors and (2) presence of pain on resisted hip adduction. Proposed methodologies to improve clinical assessment outcome measure reporting relative to adductor-related groin pain are outlined in the Minimum reporting standards on clinical assessment section.

\section{Iliopsoas-related groin pain}

Iliopsoas-related groin pain is characterised by tenderness on iliopsoas palpation. As tenderness on palpation is essential for diagnosing this entity, we recommended that, as a minimum, the presence of tenderness on palpation of the iliopsoas muscle is reported.

This clinical entity is more likely to be present if the athlete's pain is provoked by resisted hip flexion testing and/or stretching of the iliopsoas muscle. The pain on resisted hip flexion testing and/or stretching should reproduce the athlete's recognisable pain. As pain on resisted hip flexion and/or stretching of the iliopsoas is often present in athletes with iliopsoas-related groin pain, researchers should also consider quantifying and reporting the following: (1) presence or absence of the athlete's recognisable pain on resisted hip flexion and (2) presence or absence of the athlete's recognisable pain on stretching of the iliopsoas muscle. Proposed methodologies to improve clinical assessment outcome measure reporting relative to iliopsoas-related groin pain are outlined in the Minimum reporting standards on clinical assessment section.

\section{Inguinal-related groin pain}

Inguinal-related groin pain is defined as the presence of pain in the inguinal canal region and tenderness of the inguinal canal. As tenderness on palpation of the inguinal canal is essential for diagnosing this entity, we recommended that, as a minimum, the presence of tenderness on palpation of the inguinal canal is documented and reported. This clinical entity is more likely to be present if the athlete's pain is aggravated by resistance testing
Figure 3 Considerations for the reporting on clinical examination in studies on groin pain in athletes.

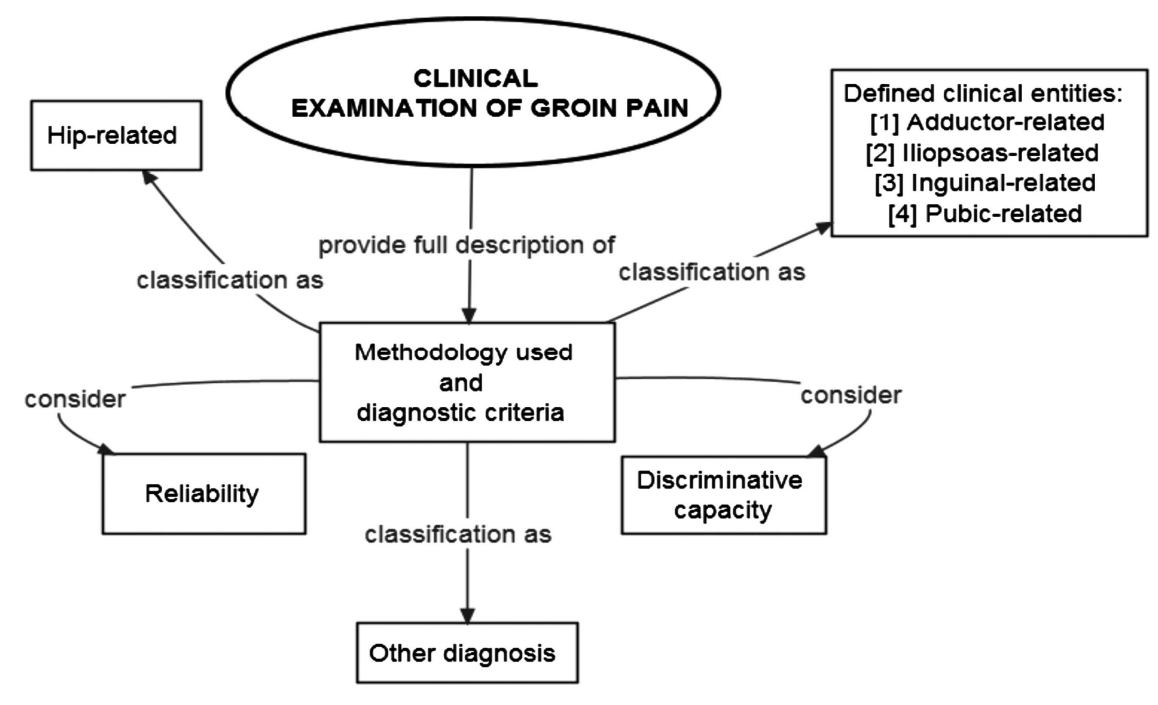


of the abdominal muscles or on Valsalva/cough/sneeze; hence, researchers should also consider reporting the following: (1) presence or absence of the athlete's recognisable pain on resistance testing of the abdominals and (2) presence or absence of the athlete's recognisable pain on Valsalva/cough/sneeze.

Pubic-related groin pain

Pubic-related groin pain is defined by localised tenderness over the pubic symphysis and immediate adjacent bone. The tenderness on palpation should reproduce the athlete's reported pain. We recommend that authors note and report the presence of tenderness on palpation immediately over the pubic symphysis and/or adjacent bone.

\section{Hip-related groin pain}

Single clinical tests characteristic of hip-related groin pain are difficult to recommend as most tests have been shown to have better sensitivity than specificity. ${ }^{18} 19$ This means that the tests are more suitable to ruling out hip-related groin pain than identifying the hip as a primary source of pain. However, we advise that during clinical examination, researchers should consider noting and reporting: (1) presence or absence of the athlete's recognisable pain on the Flexion-Adduction-Internal Rotation (FADDIR) test and (2) presence or absence of the athlete's recognisable pain on the Flexion-Abduction-External Rotation (FABER) test. Proposed methodologies to improve clinical assessment outcome measure reporting relative to hip-related groin pain are outlined in the Minimum reporting standards on clinical assessment section.

\section{Minimum reporting standards on clinical assessment}

Athletes with groin pain are often characterised by impairments as well as activity limitations and participation restrictions. These can be objectively quantified during clinical assessment. ${ }^{20}$ The objective quantification and reporting of these factors allows for a more comprehensive description of the characteristic and clinical features of groin pain in athletes. At a minimum, we recommend that researchers comprehensively describe the clinical assessment variable to be quantified, the methodology used to quantify the variable and the variable-associated descriptive statistics. Key considerations for the reporting on clinical assessment are outlined in figure 4.

In a recent systematic review by Mosler et al, ${ }^{20}$ quantification of three main factors was identified from the sports-related groin pain literature including hip muscle strength, hip range of motion, and self-reported hip and groin symptoms, in the form of patient-reported outcome measures (PROMs). Table 2 proposes some published methodologies that can aid researchers to more thoroughly describe important clinical assessment findings in athletes with groin pain.

\section{Patient-reported outcome measures}

PROMs improve the quality of assessing and reporting the outcome of treatments. Assessment of self-reported hip and groin disability can be undertaken by using specific PROMs. In a recent systematic review on the clinimetric properties of PROMs for young physically active individuals with hip and/ or groin pain, only one instrument, the Copenhagen HAGOS, existed for the assessment of individuals specifically reporting groin pain. ${ }^{32}$ The systematic review also showed that three other PROMs (Hip Outcome Score (HOS), International Hip Outcome Tool-12 (iHOT-12) and iHOT-33) existed, with all having sufficient clinimetric properties, including reliability, validity and responsiveness, to be recommended for use in individuals with hip-related pain. While HAGOS and HOS are profiles including several measurement scales measuring different aspects of impairment, activity and participation, iHOT-12 and iHOT-33 are composite scores with all domains contributing to one total score. We recommend that researchers carefully consider which aspects of impairment, activity limitations and participation restriction they want to assess in their study, as this has implications for choosing the most relevant primary outcome or aspect to be measured in the individual study. Therefore, scrutinising the content and measurement aspects in the different PROMs will aid researchers when applying a relevant measurement instrument, thus increasing their chances of quantifying the aspect of relevance and interest.

\section{Strength}

The assessment of muscle strength provides added value as a discriminative tool in observational studies or outcome measure in intervention studies. Regarding adductor-related and hip-related groin pain, we suggest that authors should consider reporting on adductor strength. The subjective assessment of adductor strength has questionable reliability; ${ }^{24}$ thus, if possible, adductor strength should be quantified more objectively. Adductor strength can be reliably assessed using hand-held dynamometry $^{28} 2933$ or by the adductor squeeze test. ${ }^{25-27}$ Regarding iliopsoas-related and hip-related groin pain, we
Figure 4 Considerations for the reporting on clinical assessment in studies on groin pain in athletes. ROM, range of motion.

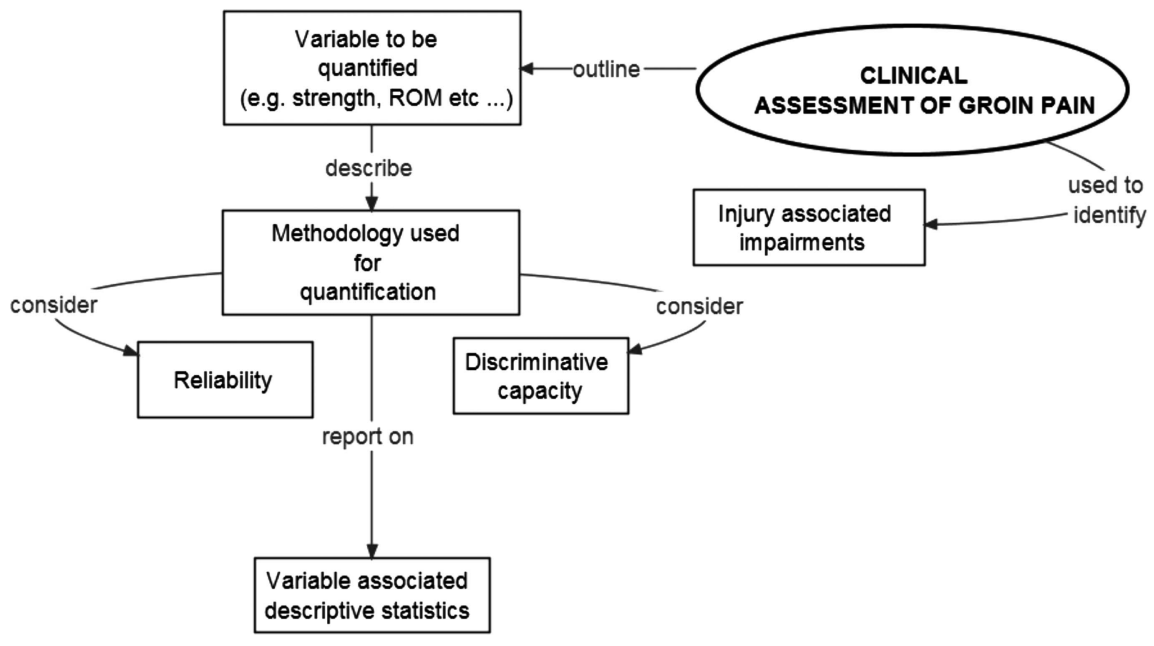


Table 2 Proposed methodologies to improve clinical assessment outcome measure reporting in studies on groin pain in athletes

\begin{tabular}{|c|c|c|}
\hline Variable & Methodology & Outcome measure(s) \\
\hline \multicolumn{3}{|l|}{ Patient-reported outcome measures } \\
\hline $\begin{array}{l}\text { Self-reported hip and groin-related } \\
\text { disability }\end{array}$ & HAGOS $^{17}$ & HAGOS subscale scores (0-100 points) \\
\hline Self-reported hip-related disability & $\mathrm{HOS}^{21}$ & HOS subscale scores $(0-100 \%)$ \\
\hline Self-reported hip-related disability & інОT-12; ${ }^{22}$ iнOT-33 $33^{23}$ & iHOT-12 score; iHOT-33 score (0-100 points) \\
\hline \multicolumn{3}{|l|}{ Strength } \\
\hline Adductor strength & $\begin{array}{l}\text { Isometric adductor strength testing } \\
\text { Adductor squeeze test }{ }^{25-27} \\
\text { Isometric adductor strength testing with } \mathrm{HHD}^{28} \\
\text { Eccentric adductor strength testing with } \mathrm{HHD}^{29}\end{array}$ & $\begin{array}{l}\text { Weak/intermediate/strong } \\
\text { Adductor squeeze test value as quantified on } \\
\text { sphygmomanometer } \\
\text { Force output on HHD (N/kg/pounds) } \\
\text { Force output on HHD (N/kg/pounds) }\end{array}$ \\
\hline Hip flexion strength & $\begin{array}{l}\text { Isometric hip flexion strength testing }{ }^{24} \\
\text { Isometric hip flexion strength testing with } \mathrm{HHD}^{28}\end{array}$ & $\begin{array}{l}\text { Weak/intermediate/strong } \\
\text { Force output on HHD (N/kg/pounds) }\end{array}$ \\
\hline \multicolumn{3}{|l|}{ Range of motion } \\
\hline Hip joint internal rotation ROM & $\begin{array}{l}\text { Passive hip joint internal rotation ROM } \\
\text { examination } 273031\end{array}$ & ROM (degrees) as quantified by a goniometer or inclinometer \\
\hline Hip joint external rotation ROM & $\begin{array}{l}\text { Passive hip joint external rotation ROM } \\
\text { examination }^{27} 3031\end{array}$ & ROM (degrees) as quantified by a goniometer or inclinometer \\
\hline
\end{tabular}

HAGOS, Copenhagen Hip and Groin Outcome Score; HHD, hand-held dynamometry; HOS, Hip Outcome Score; iHOT, International Hip Outcome Tool; ROM, range of motion.

suggest that authors should consider reporting on hip flexion strength. The subjective assessment of hip flexor strength has questionable reliability. ${ }^{24}$ Therefore, if possible, hip flexion strength should be quantified more objectively using dynamometry. ${ }^{28}$

\section{Hip joint range of motion}

We believe that hip joint range of motion assessment provides added value as a discriminative tool in observational studies or as an outcome measure in intervention studies. If relevant, internal and external hip joint rotation range of motion should be quantified in an objective and reliable manner. We suggest that authors should consider reporting on: (1) passive hip joint internal rotation range of motion in degrees and (2) passive hip joint external rotation range of motion in degrees. Hip joint range of motion can be reliably assessed using a goniometer ${ }^{31}$ or inclinometer. ${ }^{27}$

\section{Minimum reporting standards on radiology}

Of all imaging modalities, MRI has been investigated most often. The majority of studies have focused on its use for pubic and adductor-related groin pain. ${ }^{5}$ There are very few studies examining the use of MRI in iliopsoas or inguinal related groin pain in athletes. Branci et $a l^{34}$ developed a standardised MRI evaluation protocol (Copenhagen Standardized MRI protocol) for use in athletes with groin pain. The standardised MRI protocol consists of 11 predefined imaging characteristics indicative of abnormalities commonly reported in athletes with groin pain. This protocol does not assess the iliopsoas or inguinal region. Fair-to-moderate intraobserver reliability was observed for all predefined imaging characteristics with the exception of adductor tendons. This means that, at present, we are not aware of any published well-defined and reliable way of assessing the iliopsoas, inguinal region or adductor muscles using MRI.

The Copenhagen Standardized MRI protocol ${ }^{34}$ represents a starting point from which to develop a more uniform and scientific evaluation of imaging characteristics in athletes with groin pain. The adoption of the imaging techniques and terminology in this protocol will allow clinicians to compare their radiological findings in athletes with groin pain, which until now has not been possible. Further research will need to be performed to develop reliable MRI sequences and assessment techniques for the adductor, iliopsoas and inguinal regions. When reporting on MRI in research studies, a clear description of the protocol used, how abnormalities assessed for were defined and recorded, and an assessment of the reliability of the assessment, would add quality to future papers.

The use of X-rays, CT and ultrasound is not as universally well studied or established in the literature as MRI, for evaluating athletes with groin pain. While ultrasound is commonly used in clinical practice, it is under-represented in the literature. Ultrasound is well established as an accurate technique for detecting and characterising inguinal and femoral hernias, ${ }^{35-37}$ but these pathologies are not typically present clinically or radiologically in athletes with groin pain. ${ }^{5}$ Studies on ultrasound in athletes with groin pain are infrequent and the quality of those performed to date is low; therefore, it is currently difficult to recommend a minimum protocol and reporting standard for this technique. ${ }^{38}$ We encourage those reporting on ultrasound in future studies to give a clear description of the protocol used, with adequate details to allow others to reproduce the examination. The definitions of how various abnormalities are judged to be present should be clear and, again, allow for reproduction by others. The reliability and repeatability of new protocols should be examined to ensure their clinical utility.

While X-ray is frequently used in clinical practice to 'exclude bony abnormalities', most authors who have examined the role of X-ray have found bony abnormalities to be frequent. ${ }^{5} 3940$ The clinical significance of many of these findings is unknown. As is the case for MRI and ultrasound studies, describing the way findings are defined and recorded, along with the reliability of the assessment, needs to be undertaken to investigate the clinical meaning of the abnormalities commonly found on X-ray.

In summary, there are few resources available to help guide minimal reporting standards with regard to imaging. The Copenhagen Standardized MRI protocol ${ }^{34}$ provides a detailed description of the protocols used, gives a clearly defined assessment of abnormalities, and has fair-to-moderate reliability for the majority of findings. At the present time, we are not aware of detailed descriptions of reliable techniques for any imaging 
modality to assess for abnormalities of the adductors, iliopsoas or inguinal region (with the exception of hernias). Future studies should aim to develop these techniques, so that the clinical relevance of the abnormalities commonly found on imaging can be examined. Key considerations for the reporting on radiology are outlined in figure 5 .

\section{DISCUSSION}

Consensus statements in sports medicine and sports physiotherapy are needed relative to study type, sport and injury under investigation. In this paper, we propose minimum reporting standards to outline important criteria that should be considered to include when undertaking and disseminating research on groin pain in athletes. Poor design and reporting of studies undermines their clinical usefulness, and recent reviews illustrate the predominance of heterogeneous low-quality studies on groin pain in athletes. ${ }^{3} 5$ As such, it is clear that current peer-review processes are failing in this respect. Therefore, we feel that these recommendations regarding minimum reporting standards are justified and useful.

The concepts of defined terminologies and minimum reporting standards are not new in sports physiotherapy and sports medicine. Inconsistencies in research results have been noted in the area of ankle joint sprain and CAI. ${ }^{41}$ Delahunt et $a l^{41}$ devised a set of operational definitions and minimum reporting standards related to ankle joint sprain and CAI. These were recently endorsed and expanded on in a position statement of the International Ankle Consortium. ${ }^{6} 78$ Encouragingly, recent papers published in the area have started to implement the recommendations of the International Ankle Consortium. ${ }^{42-44}$

Our minimum reporting standards on study methodology advocate the use of established published guidelines to improve methodological design and reporting. Clinical trials should have an associated protocol that documents the rationale for the study, proposed methodologies, as well as organisational and ethical considerations. The SPIRIT 2013 statement consists of a 33 -item checklist of minimum recommended protocol items that can be used by researchers preparing the full protocol of a clinical trial. ${ }^{12}$ Properly designed and executed RCTs provide a reliable source of evidence regarding the effectiveness of healthcare interventions. Critical appraisal of the quality of RCTs is only possible if the design, conduct and analysis of the trial are described in an unambiguous, transparent manner. ${ }^{9}$ The reporting of RCTs is often incomplete. ${ }^{45}{ }^{46}$ The CONSORT 2010 statement provides researchers with a checklist and flow diagram that can be used for reporting a RCT, and it facilitates easier critical appraisal and interpretation of the study. ${ }^{9}$ The TREND statement provides an alternative for the standardised and transparent reporting of non-randomised intervention research. ${ }^{10}$ The credibility of observational studies depends on the ability to critically assess the strengths and weaknesses of the study design, conduct and analysis. As such, the STROBE statement provides a checklist of 22 items that should be included in reports of cohort, case-control and cross-sectional studies. ${ }^{11}$ The evaluation of healthcare interventions is vitally important, yet it has been reported that the quality of descriptions of interventions in the published literature is quite poor. ${ }^{16}$ Furthermore, without an adequate, transparent description of the intervention, it is difficult for other researchers to replicate or build on research findings. The TIDieR checklist provides an extension of item 5 of the CONSORT 2010 statement and item 11 of the SPIRIT 2013 statement, with the aim of encouraging authors to describe interventions in sufficient detail to allow their direct replication. ${ }^{16}$

It is apparent from the groin pain literature that minimum reporting standards on study methodology, study participants and injury history, clinical examination, clinical assessment and radiology are all necessary. Our outlined minimum reporting standards on study participants and injury history will facilitate a comprehensive description of participants enrolled in research studies. Clearly defined information on study participants and their injury history will enable readers to interpret the applicability and relevance of research findings to their clinical practice. Our recommended minimum reporting standards on clinical examination are aligned with the "Doha agreement meeting on terminology and definitions in groin pain in athletes". ${ }^{4}$ Additionally, for clinical assessment of athletes with groin pain, we provide guidance on variables to be quantified, methodologies for quantifying these variables and associated appropriate outcome measures. Such guidance may allow for a more objective structured clinical examination, with appropriate reporting of quantifiable meaningful outcome measures.

\section{CONCLUSION}

The high prevalence and incidence of groin pain in athletes supports the necessity for continued methodologically rigorous research on prevention and treatment. Based on collective expertise, and the best available evidence, we encourage the use of these minimum reporting standards in future research. In the coming years, it will be necessary to assess whether authors of
Figure 5 Considerations for the reporting on radiology in studies on groin pain in athletes.

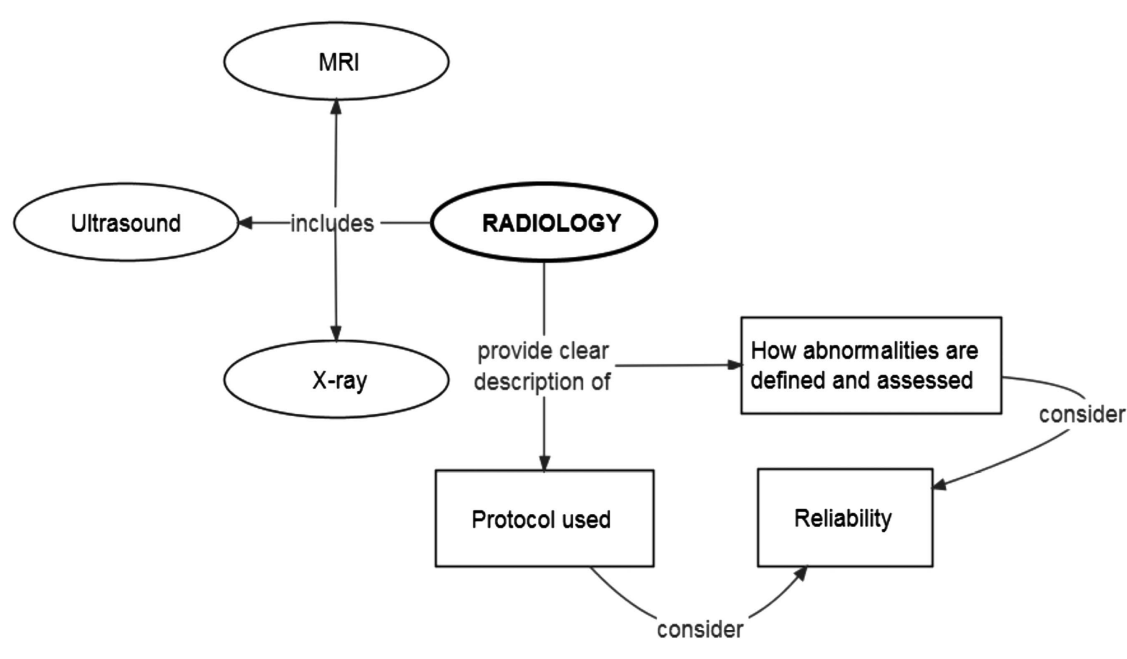


research on groin pain in athletes adopt and implement our recommendations; and whether there is a concomitant improvement in study methodological quality and specific reporting on clinical examination, clinical assessment and radiology.

\section{What is already known?}

Heterogeneous studies with low methodological quality dominate research related to groin pain in athletes.

\section{What are the new findings?}

- We propose a set of minimum reporting standards based on best available evidence to be utilised in future research on groin pain in athletes.

- Minimum reporting standards are provided in relation to: (1) study methodology, (2) study participants and injury history, (3) clinical examination, (4) clinical assessment and (5) radiology.

Contributors ED, PH, KMK, KT and AW developed the idea for the manuscript. All authors contributed to the writing, revision and final approval of the manuscript.

Competing interests None declared.

Provenance and peer review Not commissioned; externally peer reviewed.

Open Access This is an Open Access article distributed in accordance with the Creative Commons Attribution Non Commercial (CC BY-NC 4.0) license, which permits others to distribute, remix, adapt, build upon this work non-commercially, and license their derivative works on different terms, provided the original work is properly cited and the use is non-commercial. See: http://creativecommons.org/ licenses/by-nc/4.0/

\section{REFERENCES}

1 Orchard JW. Men at higher risk of groin injuries in elite team sports: a systematic review. Br J Sports Med 2015:49:798-802.

2 Waldén $M$, Hägglund $M$, Ekstrand J. The epidemiology of groin injury in senior football: a systematic review of prospective studies. Br J Sports Med 2015;49: 792-7.

3 Serner $\mathrm{A}$, van Eijck $\mathrm{CH}$, Beumer BR, et al. Study quality on groin injury management remains low: a systematic review on treatment of groin pain in athletes. Br J Sports Med 2015;49:813.

4 Weir $A$, Brukner $P$, Delahunt $E$, et al. Doha agreement meeting on terminology and definitions in groin pain in athletes. Br J Sports Med 2015;49:768-74.

5 Branci S, Thorborg K, Nielsen MB, et al. Radiological findings in symphyseal and adductor-related groin pain in athletes: a critical review of the literature. $\mathrm{Br} J$ Sports Med 2013:47:611-19.

6 Gribble PA, Delahunt E, Bleakley C, et al. Selection criteria for patients with chronic ankle instability in controlled research: a position statement of the International Ankle Consortium. Br J Sports Med 2014;48:1014-18.

7 Gribble PA, Delahunt E, Bleakley C, et al. Selection criteria for patients with chronic ankle instability in controlled research: a position statement of the International Ankle Consortium. J Athl Train 2014:49:121-7.

8 Gribble PA, Delahunt E, Bleakley C, et al. Selection criteria for patients with chronic ankle instability in controlled research: a position statement of the International Ankle Consortium. J Orthop Sports Phys Ther 2013:43:585-91.

9 Moher D, Hopewell S, Schulz KF, et al. CONSORT 2010 explanation and elaboration: updated guidelines for reporting parallel group randomised trials. BMJ 2010;340:c869

10 Des Jarlais DC, Lyles C, Crepaz, et al. TREND Group Improving the reporting quality of nonrandomized evaluations of behavioral and public health interventions: the TREND statement. Am J Public Health 2004;94:361-6.

11 von Elm E, Altman DG, Egger M, et al. Strengthening the Reporting of Observational Studies in Epidemiology (STROBE) statement: guidelines for reporting observational studies. BMJ 2007:335:806-8.
12 Chan AW, Tetzlaff JM, Gøtzsche PC, et al. SPIRIT 2013 explanation and elaboration: guidance for protocols of clinical trials. BMJ 2013;346:e7586.

13 http://www.icmje.org/recommendations/browse/publishing-and-editorial-issues/ clinical-trial-registration.html (accessed Apr 2015).

14 http://www.who.int/ictrp/en/ (accessed Apr 2015)

15 https://clinicaltrials.gov (accessed Apr 2015).

16 Hoffmann TC, Glasziou PP, Boutron I, et al. Better reporting of interventions: template for intervention description and replication (TIDieR) checklist and guide. BMJ 2014;348:g1687.

17 Thorborg K, Hölmich P, Christensen R, et al. The Copenhagen Hip and Groin Outcome Score (HAGOS): development and validation according to the COSMIN checklist. Br J Sports Med 2011;45:478-91.

18 Reiman MP, Thorborg K. Clinical examination and physical assessment of hip joint-related pain in athletes. Int J Sports Phys Ther 2014;9:737-55.

19 Reiman MP, Goode AP, Hegedus EJ, et al. Diagnostic accuracy of clinical tests of the hip: a systematic review with meta-analysis. $\mathrm{Br} J$ Sports $\mathrm{Med}$ 2013:47:893-902.

20 Mosler AB, Agricola R, Weir A, et al. Factors differentiating athletes with and without hip and groin pain - a systematic review and meta-analysis. $\mathrm{Br} J$ Sports Med 2015;49:810

21 Martin RL, Kelly BT, Philippon MJ. Evidence of validity for the hip outcome score. Arthroscopy 2006;22:1304-11.

22 Griffin DR, Parsons N, Mohtadi NG, et al. A short version of the International Hip Outcome Tool (iHOT-12) for use in routine clinical practice. Arthroscopy 2012;28:611-16.

23 Mohtadi NG, Griffin DR, Pedersen ME, et al. The development and validation of a self-administered quality-of-life outcome measure for young, active patients with symptomatic hip disease: the International Hip Outcome Tool (iHOT-33). Arthroscopy 2012;28:595-605.

24 Hölmich P, Hölmich LR, Bjerg AM. Clinical examination of athletes with groin pain: an intraobserver and interobserver reliability study. $\mathrm{Br} J$ Sports $\mathrm{Med}$ 2004;38:446-51.

25 Delahunt $\mathrm{E}, \mathrm{McEntee} \mathrm{BL}$, Kennelly $\mathrm{C}$, et al. Intrarater reliability of the adductor squeeze test in gaelic games athletes. J Athl Train 2011:46:241-5.

26 Delahunt E, Kennelly C, McEntee BL, et al. The thigh adductor squeeze test: $45^{\circ}$ of hip flexion as the optimal test position for eliciting adductor muscle activity and maximum pressure values. Man Ther 2011:16:476-80.

27 Malliaras $\mathrm{P}$, Hogan A, Nawrocki A, et al. Hip flexibility and strength measures: reliability and association with athletic groin pain. Br J Sports Med 2009:43:739-44.

28 Thorborg K, Petersen J, Magnusson SP, et al. Clinical assessment of hip strength using a hand-held dynamometer is reliable. Scand J Med Sci Sports 2010:20:493-501.

29 Thorborg K, Couppé C, Petersen J, et al. Eccentric hip adduction and abduction strength in elite soccer players and matched controls: a cross-sectional study. $\mathrm{Br} \mathrm{J}$ Sports Med 2011:45:10-13.

30 Nevin F, Delahunt E. Adductor squeeze test values and hip joint range of motion in Gaelic football athletes with longstanding groin pain. I Sci Med Sport 2014;17:155-9.

31 Nussbaumer S, Leunig M, Glatthorn JF, et al. Validity and test-retest reliability of manual goniometers for measuring passive hip range of motion in femoroacetabular impingement patients. BMC Musculoskelet Disord 2010;11:194.

32 Thorborg K, Tijssen M, Habets B, et al. Patient-Reported Outcome (PRO) questionnaires for young to middle-aged adults with hip and groin disability: a systematic review of the clinimetric evidence. $\mathrm{Br}$ J Sports Med 2015;49:812

33 Fulcher ML, Hanna CM, Raina Elley C. Reliability of handheld dynamometry in assessment of hip strength in adult male football players. I Sci Med Sport 2010;13:80-4.

34 Branci $\mathrm{S}$, Thorborg $\mathrm{K}$, Bech $\mathrm{BH}$, et al. MRI findings in soccer players with longstanding adductor-related groin pain and asymptomatic controls. $\mathrm{Br} J$ Sports Med 2015:49:681-91.

35 Pawlak M, Niebuhr H, Bury K. Dynamic inguinal ultrasound: a diagnostic tool for hernia surgeons. Hernia 2015. Published Online First.

36 Kim B, Robinson $\mathrm{P}$, Modi $\mathrm{H}$, et al. Evaluation of the usage and influence of groin ultrasound in primary and secondary healthcare settings. Hernia 2014. Published Online First.

37 Djuric-Stefanovic A, Saranovic D, Ivanovic A, et al. The accuracy of ultrasonography in classification of groin hernias according to the criteria of the unified classification system. Hernia 2008;12:395-400.

38 Robinson P, Grainger AJ, Hensor EM, et al. Do MRI and ultrasound of the anterior pelvis correlate with, or predict, young football players' clinical findings? A 4-year prospective study of elite academy soccer players. Br J Sports Med 2015:49:176-82

39 Weir A, de Vos RJ, Moen M. Prevalence of radiological signs of femoroacetabular impingement in patients presenting with long-standing adductor-related groin pain. Br J Sports Med 2011:45:6-9. 
40 Hölmich $\mathrm{P}$, Thorborg K, Nyvold P, et al. Does bony hip morphology affect the outcome of treatment for patients with adductor-related groin pain? Outcome 10 years after baseline assessment. Br J Sports Med 2014;48:1240-4.

41 Delahunt E, Coughlan GF, Caulfield B, et al. Inclusion criteria when investigating insufficiencies in chronic ankle instability. Med Sci Sports Exerc 2010:42:2106-21.

42 Kim KM, Hart JM, Saliba SA, et al. Effects of focal ankle joint cooling on unipedal static balance in individuals with and without chronic ankle instability. Gait Posture 2015;41:282-7.
43 Levin 0 , Vanwanseele B, Thijsen JR, et al. Proactive and reactive neuromuscular control in subjects with chronic ankle instability: evidence from a pilot study on landing. Gait Posture 2015;41:106-11.

44 Terada M, Bowker S, Thomas AC, et al. Alterations in stride-to-stride variability during walking in individuals with chronic ankle instability. Hum Mov Sci 2014;40C:154-62.

45 Adetugbo K, Williams H. How well are randomized controlled trials reported in the dermatology literature? Arch Dermatol 2000;136:381-5.

46 Kjaergard LL, Nikolova D, Gluud C. Randomized clinical trials in HEPATOLOGY: predictors of quality. Hepatology 1999;30:1134-8. 